\title{
Vagal activity and oxygen saturation response to hypoxia: Effects of aerobic fitness and rating of hypoxia tolerance
}

\author{
Tomášs Macoun', Michal Botek ${ }^{1}$, Jakub Krejčí1,*, and Andrew J. McKune ${ }^{2,3}$ \\ ${ }^{I}$ Department of Natural Sciences in Kinanthropology, Faculty of Physical Culture, Palacký University Olomouc, Olomouc, \\ Czech Republic; ${ }^{2}$ Discipline of Sport and Exercise Science, UC - Research Institute for Sport and Exercise, Faculty of \\ Health, University of Canberra, ACT, Australia; and ${ }^{3}$ Discipline of Biokinetics, Exercise and Leisure Sciences, School of \\ Health Sciences, University of KwaZulu-Natal, Durban, South Africa
}

Copyright: (c) 2017 T. Macoun et al. This is an open access article licensed under the Creative Commons Attribution License (http://creativecommons.org/licenses/by/4.0/).

\begin{abstract}
Background: A reduction in the inspired oxygen fraction $\left(\mathrm{FiO}_{2}\right)$ induces a decline in arterial oxygen saturation $\left(\mathrm{SpO}_{2}\right)$ and changes of heart rate variability (HRV). It has been shown that $\mathrm{SpO}_{2}$ and $\mathrm{HRV}$ responses to similar levels of acute normobaric hypoxia are inter-individual variable. Variable response may be influenced by normoxia reached maximal oxygen uptake $\left(\mathrm{VO}_{2} \max \right)$ value. Objective: The primary aim was to assess $\mathrm{HRV}$ and the $\mathrm{SpO}_{2}$ response to hypoxia, and examine the association with normoxic $\mathrm{VO}_{2}$ max. Methods: Supine $\mathrm{HRV}$ and $\mathrm{SpO}_{2}$ were monitored during normobaric hypoxia $\left(\mathrm{FiO}_{2}=9.6 \%\right)$ for 10 minutes in 28 subjects, aged $23.7 \pm 1.7$ years. HRV was evaluated by using both spectral and time domain HRV analysis. Low frequency (LF, 0.05-0.15 Hz) and high frequency (HF, $0.15-0.50 \mathrm{~Hz}$ ) power together with square root of the mean of the squares of the successive differences (rMSSD) were calculated and transformed by natural logarithm $(\mathrm{Ln})$. Based on the $\mathrm{SpO}_{2}$ in hypoxia, subjects were divided into Resistant (RG, $\mathrm{SpO}_{2} \geq 70.9 \%, n=14$ ) and Sensitive ( $\mathrm{SG}, \mathrm{SpO}_{2}<70.9 \%, n=14$ ) groups. Perceived hypoxia tolerance was self-scored on a 4-level scale. Results: $\mathrm{VO}_{2}$ max was higher in $\mathrm{SG}\left(62.4 \pm 7.2 \mathrm{ml} \cdot \mathrm{kg}^{-1} \cdot \mathrm{min}^{-1}\right)$ compared with $\mathrm{RG}\left(55.5 \pm 7.1 \mathrm{ml} \cdot \mathrm{kg}^{-1} \cdot \mathrm{min}^{-1}, p=.017, d=0.97\right)$. A significant relationship $(r=-.45, p=.017)$ between hypoxicnormoxic difference in $\mathrm{SpO}_{2}$ and normoxic $\mathrm{VO}_{2}$ max level was found. Vagal activity (Ln rMSSD) was significantly decreased (SG: $p<.001, d=2.64$; RG: $p<.001, d=1.22$ ), while sympathetic activity (Ln LF/HF) was relatively increased $(p<.001, d=-1.40)$ in only the SG during hypoxia. Conclusions: Results show that subjects with a higher aerobic capacity exhibited a greater decline in $\mathrm{SpO}_{2}$, accompanied by greater autonomic cardiac disturbances during hypoxia. The $\mathrm{SpO}_{2}$ reduction was associated with perceived hypoxia comfort/discomfort. The hypoxia discomfort state was accompanied by a greater withdrawal in cardiac vagal activity.
\end{abstract}

Keywords: simulated altitude, haemoglobin desaturation, heart rate variability, maximal oxygen uptake, acute mountain sickness

\section{Introduction}

In general, a reduction in the inspired oxygen fraction $\left(\mathrm{FiO}_{2}\right)$ induces a decline in oxygen saturation $\left(\mathrm{SpO}_{2}\right)$ (Iwasaki et al., 2006; Pighin et al., 2012) and causes homeostatic impairment due to systemic hypoxia (Mizuno et al., 1990; Ventura et al., 2003). The acute response of the $\mathrm{O}_{2}$ transport system to hypoxia is mediated through changes in autonomic cardiac regulation

\footnotetext{
* Address for correspondence: Jakub Krejčí, Department of Natural Sciences in Kinanthropology, Faculty of Physical Culture, Palacký University Olomouc, třída Míru 117, 77111 Olomouc, Czech Republic. E-mail: jakub.krejci@upol.cz
}

and a neurohumoral mechanism that lead e.g. to an increase in heart rate (HR), cardiac output, and/or to elevation in minute ventilation (Åstrand, Rodahl, Dahl, \& Strømme, 2003). A number of studies, which used different durations and levels of normobaric hypoxia, have focused on the autonomic nervous system (ANS) response using heart rate variability (HRV) assessment and have reported vagal activity withdrawal, and a relative increase in sympathetic activity (Al Haddad, Mendez-Villanueva, Bourdon, \& Buchheit, 2012; Narkiewicz et al., 2006; Wille et al., 2012). The spectral analysis (SA) of HRV is a non-invasive tool to determine changes in autonomic cardiac activity (Akselrod et al., 1981). High frequency power (HF), reflects the influence of 
the vagal outflow and vagally related respiratory modulation of the heart known as respiratory sinus arrhythmia (RSA) (Task Force of the European Society of Cardiology and the North American Society of Pacing and Electrophysiology, 1996; Yasuma \& Hayano, 2004). Low frequency power (LF) is considered the dominant indicator of sympathetic activity by Ursino and Magosso (2003) while other authors understand the LF to be an indicator of both sympathetic and vagal activity together with baroreceptors activity (Malik \& Camm, 1993; Malliani, Lombardi, \& Pagani, 1994; Zygmunt \& Stanczyk, 2010). In addition, LF/HF ratio reflects the sympathovagal balance (Malliani, Pagani, Lombardi, \& Cerutti, 1991). HRV may be also evaluated via using a time domain analysis (Buchheit, 2014).

Some studies have revealed that a similar level of short-term acute normobaric hypoxia induces individual differences in the magnitude of $\mathrm{SpO}_{2}$ response that consequently are related to variable changes in autonomic cardiac regulation (Bobyleva \& Glazachev, 2007; Botek, Krejčí, De Smet, Gába, \& McKune, 2015). Importantly, the $\mathrm{SpO}_{2}$ response to hypoxia at rest (Burtscher, Flatz, \& Faulhaber, 2004) and the combination of rest and exercise (Karinen, Peltonen, Kähönen, \& Tikkanen, 2010; Rathat, Richalet, Herry, \& Larmignat, 1992) has been suggested by some authors to be a promising marker of susceptibility to acute mountain sickness (AMS) development, while others have reported opposite findings (Wille et al., 2012; O'Connor, Dubowitz, \& Bickler, 2004). For example, subjects that manifested AMS symptoms exhibited both a greater decline in $\mathrm{SpO}_{2}$ in hypoxia and a higher maximal oxygen uptake $\left(\mathrm{VO}_{2} \max \right)$ compared with subjects who were free from AMS symptoms (Karinen et al., 2010). Previous research also reported that $\mathrm{VO}_{2} \max$ may have a negative influence on the $\mathrm{SpO}_{2}$ response to simulated altitude (2500-4600 m) at rest (Woorons et al., 2007) but particularly during moderate exercise intensity. Considering the above mentioned findings, we propose that differences in normoxic $\mathrm{VO}_{2}$ max may contribute to the individual variation in $\mathrm{SpO}_{2}$ during hypoxic exposure, and consequently in the autonomic cardiac response to acute normobaric hypoxia at rest. Therefore, the primary aim of this study was to assess the $\mathrm{SpO}_{2}$ response to hypoxia $\left(\mathrm{FiO}_{2}=9.6 \%\right)$ and the concomitant changes in autonomic cardiac regulation during resting conditions. The secondary aim was to determine the relationship between changes in $\mathrm{SpO}_{2}$ response to hypoxia in subjects with different cardiorespiratory performance, and further to assess, whether 10 minutes of hypoxia may have an impact on subjective perceived discomfort in healthy male subjects.

\section{Methods}

\section{Participants}

The testing group consisted of 28 healthy, physically active, non-smoking, male volunteers (aged $23.7 \pm 1.7$ years, weight $78.4 \pm 7.9 \mathrm{~kg}$, height $180.3 \pm 7.2 \mathrm{~cm}$ ). The study was approved by the Institutional Research Ethics Committee and conformed to the recommendations from the Declaration of Helsinki. Written informed consent was obtained from each participant. Participants underwent preliminary medical screening to identify cardiovascular and pulmonary conditions that would exclude them from the study. The exclusion criteria included pathological changes in cardiac rhythm, hypertension, smoking and acute respiratory disease. The volunteers were free of medical complications taking no medication or dietary supplements which could impact the results of the test. The subjects had not been exposed to hypoxic environments for at least the previous two years.

\section{Maximal oxygen uptake determination}

$\mathrm{VO}_{2}$ max, as a global indicator of physical fitness, and maximum heart rate (HRmax), were measured in normoxia during an incremental running test on the treadmill (Valiant Plus, Lode, Groningen, Netherlands). The protocol consisted of a four minute warm-up ( 2 minutes at $8 \mathrm{~km} \cdot \mathrm{h}^{-1}$, with $0 \%$ elevation and then two minutes at the same speed at $5 \%$ elevation) followed by an increase in speed to $10 \mathrm{~km} \cdot \mathrm{h}^{-1}$ at $5 \%$ elevation for 1 minute. From this point, at each minute, the speed was increased by $1 \mathrm{~km} \cdot \mathrm{h}^{-1}$, keeping elevation the same, up to $16 \mathrm{~km} \cdot \mathrm{h}^{-1}$. Then the speed was maintained and only the elevation increased by $2.5 \%$ per minute until exhaustion. Ventilation and gas exchange were recorded continuously (breath by breath) with 30 second averaging and analysed by Blue Cherry 1.2 software (Geratherm Respiratory, Bad Kissingen, Germany). The criteria for attaining $\mathrm{VO}_{2} \max$ was defined as reaching one of the following criteria: a) respiratory exchange ratio of > 1.11 (Howley, Bassett, \& Welch, 1995); b) $\mathrm{VO}_{2}$ plateau (defined as no increase in $\mathrm{VO}_{2}$ in response to an increase in work rate; Midgley, McNaughton, Polman, \& Marchant, 2007). $\mathrm{VO}_{2}$ max was considered the highest $\mathrm{VO}_{2}$ value in the final 30 seconds of the test (Millet et al., 2003). HR response was measured continuously using WearLink chest strap (Polar, Kempele, Finland).

\section{Hypoxic experiment}

One week after $\mathrm{VO}_{2}$ max determination (Figure 1), the hypoxic experiment was performed between 8:00 and 11:00 a.m. in a laboratory under standardized conditions (temperature 22 to $24{ }^{\circ} \mathrm{C}$, relative humidity $\leq 60 \%$ ). Acoustic and visual stimuli were also 


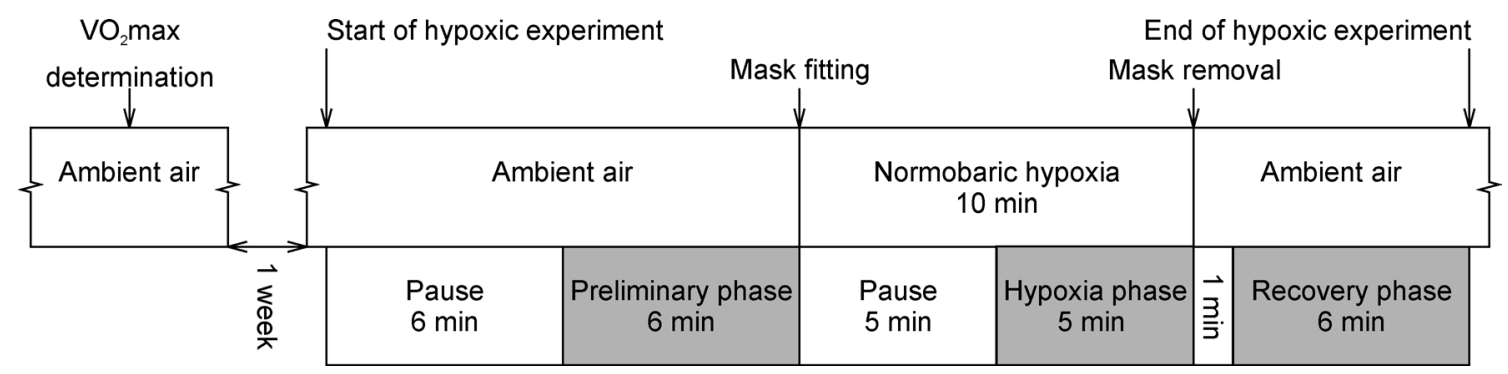

Figure 1. Course of hypoxic experimental protocol. Grey coloured phases were intended for $\mathrm{SpO}_{2}$ and ECG recording. Pauses were used to skip the transitory phase and to be able to assume stationarity of the data.

controlled to reduce their effects on HRV (Hori et al., 2005). Subjects were required to avoid eating, drinking coffee, tea and/or any substance affecting the ANS activity for at least two hours before the hypoxic experiment. In addition, they were asked to avoid vigorous physical activity and alcohol for 48 hours before the hypoxic experiment.

The hypoxic experiment proceeded as follows (Figure 1): the subjects first breathed ambient air without a breathing mask. Each subject lay supine for 6 minutes to skip the transitory phase and to be able to assume stationarity of the data (Task Force of the European Society of Cardiology and the North American Society of Pacing and Electrophysiology, 1996). After this standardization pause, $\mathrm{SpO}_{2}$ and electrocardiogram (ECG) data was recorded for 6 minutes and was used for calculation of "Preliminary phase" variables. When the preliminary recording was done, a research assistant fitted a face mask and the subject started to breathe air with a reduced $\mathrm{O}_{2}$ concentration. The first 5 minutes of hypoxia served as a standardization pause and last 5 minutes was used for recording and calculation of "Hypoxia phase" variables. When 10 minutes of hypoxia elapsed, the mask was removed and the subject again breathed ambient air. One minute was skipped as standardization pause (Buchheit et al., 2004) and 6 minute data recording followed. This data was used for calculation of "Recovery phase" variables.

The altitude of the laboratory was $260 \mathrm{~m}$ above sea level. The normobaric hypoxic environment $\left(\mathrm{FiO}_{2}=9.6 \%\right.$, simulated altitude of $\left.6200 \mathrm{~m}\right)$ was created using the MAG-10 system (Higher Peak, Boston, MA, USA). The given normobaric hypoxia level has been widely used in previous studies for intermittent hypoxia exposure (Millet, Roels, Schmitt, Woorons, \& Richalet, 2010), and/or to determine effect of intermittent hypoxia training to autonomic cardiac regulation (Bobyleva \& Glazachev, 2007).

\section{Oxygen saturation measurement}

$\mathrm{SpO}_{2}$ was measured continuously throughout the experimental protocol using a Nonin Avant 4000 (Nonin Medical, Minneapolis, MN, USA) pulse oximeter set on the right index finger. The oximeter served as a data collection device as well as a safety measure to ensure subject safety during hypoxia. The change in $\mathrm{SpO}_{2}$ between the Hypoxia phase and Preliminary phase was calculated as $\Delta \mathrm{SpO}_{2}=\mathrm{SpO}_{2 \text { Hypoxia }}-\mathrm{SpO}_{2 \text { Preliminary. }}$.

\section{Heart rate variability analysis}

To determine the breathing frequency (BF), HR, and HRV variables, the ECG was recorded at a sampling frequency of $1000 \mathrm{~Hz}$ using DiANS PF8 diagnostic system (Dimea Group, Olomouc, Czech Republic). The system includes a chest strap, unit for recording and transmitting of ECG data, and a receiver connected to a personal computer with the special software. The ECG record was examined, and all ectopic beats and any artefacts were manually removed. The BF was determined based on changes in amplitude of QRS complex caused by respiratory movements (Salinger et al., 2005). A set of 300 artefact-free RR intervals was obtained from each measurement phase (Preliminary, Hypoxia, and Recovery). A SA HRV method was used to assess the cardiac autonomic activity and was performed using the Fast Fourier Transform (Task Force of the European Society of Cardiology and the North American Society of Pacing and Electrophysiology, 1996). Spectral powers were calculated in the bands as follows: $\mathrm{LF}$ from 0.05 to $0.15 \mathrm{~Hz}$ and $\mathrm{HF}$ from 0.15 to $0.50 \mathrm{~Hz}$. Additionally, LF/HF ratio and a time domain variable, the square root of the mean of the squares of the successive differences (rMSSD) was calculated.

From a methodological perspective, the influence of breathing patterns and tidal volume on the HRV components is well described (Brown, Beightol, Koh, \& Eckberg, 1993; Hirsch \& Bishop, 1981) where a decrease in $\mathrm{BF}$ and increased tidal volume cause an increase in the $\mathrm{HF}$ component. However, a $\mathrm{BF}<9$ 
breaths per minute may lead to an artificial increase in the LF with concomitant changes in the LF/HF ratio due to an RSA peak shift from HF into the LF. This could be a limitation in terms of interpreting both the vagal and the sympathetic contribution to the sinoatrial node activity (Sasaki \& Maruyama, 2014; Vlčková et al., 2005). To avoid the potential methodological issue of $\mathrm{BF}, \mathrm{HRV}$ is frequently measured under paced breathing conditions (Botek et al., 2015; Roche et al., 2002). However, it has been reported that paced breathing may increase sympathetic activity (Patwardhan, Vallurupalli, Evans, Bruce, \& Knapp, 1995). Therefore, in the present study we used spontaneous breathing throughout the experimental protocol. In this context, Penttilä et al. (2001) demonstrated that when spontaneous breathing is employed whilst measuring HRV, the time domain variable, rMSSD, is more resistant to altered $\mathrm{BF}$ and therefore seems to be a more accurate index of vagal activity than $\mathrm{HF}$.

\section{Subjective rating of comfort/discomfort during hypoxia}

In order to examine the subjective score of well-being during hypoxia, we created a 4-level scale, adapted from Roach et al. (2000). Immediately before the end of the 10 minutes of hypoxia, the subjects were asked to score their hypoxia subjective state on a 4-level scale. If the subjects experienced nausea or vomiting, headache, weakness during hypoxia, exposure to the hypoxia was stopped prematurely and this condition would be scored as a 0 level (severe incapacitating discomfort). None of the subjects experienced this level in the study. Level 1 represented moderate discomfort, level 2 mild discomfort, and level 3 no discomfort. Based on these subjective scores the subjects were divided into two groups: hypoxia discomfort group (HDG, ratings 1 and 2, $n=14$,) and hypoxia comfort group (HCG, rating $3, n=14$ ).

\section{Statistical analysis}

All data were checked for normality using the Kolmogorov-Smirnov test and are expressed as the mean \pm standard deviation. Skewed probability distributions of $\mathrm{LF}, \mathrm{HF}, \mathrm{LF} / \mathrm{HF}$, and rMSSD variables were corrected applying a natural logarithm (Ln). According to previously published studies (Bobyleva \& Glazachev, 2007; Botek et al., 2015) subjects were divided into two groups based on the median $\mathrm{SpO}_{2}$ value during the hypoxic phase $(70.9 \%)$. The subjects with $\mathrm{SpO}_{2} \geq 70.9 \%$ were assigned to the Resistant group (RG, range 70.9-86.7\%, $n=14)$. The subjects with $\mathrm{SpO}_{2}<70.9 \%$ were assigned to the Sensitive group (SG, range 58.4-70.9\%, $n=14$ ). A two-way analysis of variance (ANOVA) for repeated measures was used to evaluate the effect of hypoxia on selected variables. When the ANOVA revealed a significant effect, multiple comparisons via the Fisher's
LSD post-hoc test were performed. A two-sample $t$-test was used to evaluate differences in selected variables between groups (SG vs RG, and HDG vs HCG). For all tests, statistical significance was set at $\alpha<0.05$.

Effect size was calculated as standardized mean difference according the formula (Fritz, Morris, \& Richler, 2012) $d=(m 1-m 2) / S D_{\mathrm{p}}$ where $m 1$ and $m 2$ are means to compare. Pooled standard deviation was calculated as follows (Fritz et al., 2012)

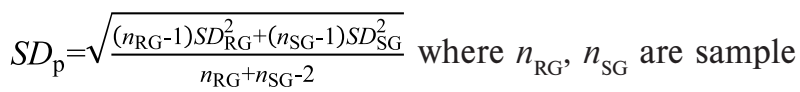
sizes and $S D_{\mathrm{RG}}, S D_{\mathrm{SG}}$ are standard deviations of $\mathrm{RG}$, $\mathrm{SG}$, respectively. When performing multiple comparisons (Figure 2), $\mathrm{SD}_{\mathrm{RG}}$ and $\mathrm{SD}_{\mathrm{SG}}$ were taken from Preliminary phase only as recommended by (Morris \& DeShon, 2002) because $S D$ of Hypoxia and Recovery phases can be influenced by hypoxia exposure. Because $S D_{\mathrm{p}}=1.1 \%$ calculated for $\mathrm{SpO}_{2}$ was below the accuracy of oximeter sensor expressed as $S D=2 \%$ (Nonin Avant 4000 datasheet), we set $S D_{\mathrm{p}}=2 \%$ for calculating effect size of $\mathrm{SpO}_{2}$ changes. The following threshold values for effect size were adopted (Hopkins, Marshall, Batterham, \& Hanin, 2009): < 0.2 (trivial), $\geq 0.2$ (small), $\geq 0.6$ (moderate),$\geq 1.2$ (large),$\geq 2.0$ (very large),$\geq 4.0$ (extremely large).

The Pearson's correlation coefficient $(r)$ was calculated to assess the relationships between $\Delta \mathrm{SpO}_{2}$ and different variables. The magnitude of $r$ was interpreted according following thresholds (Hopkins et al., 2009): $<0.1$ (trivial), $\geq 0.1$ (small), $\geq 0.3$ (moderate), $\geq 0.5$ (large), $\geq 0.7$ (very large), and $\geq 0.9$ (extremely large). Statistical analysis was performed using STATISTICA 12.0 (StatSoft, Tulsa, OK, USA) and MATLAB 8.4 (MathWorks, Natick, MA, USA).

\section{Results}

Means and standard deviations of $\mathrm{SpO}_{2}, \mathrm{HR}, \mathrm{HRV}$ variables, and BF during Preliminary, Hypoxia, and Recovery phases are provided in Table 1. Statistical significances and effect sizes of changes between phases and also between RG and SG are depicted in Figure 2.

Correlation analyses (Figure 3) showed significant associations between $\Delta \mathrm{SpO}_{2}$ and $\Delta \mathrm{Ln} \mathrm{HF}(r=.54$, large effect, $p=.003$ ) and between $\Delta \mathrm{SpO}_{2}$ and $\Delta \mathrm{Ln}$ rMSSD $(r=.64$, large effect, $p<.001)$. A significant relationship was also found for changes in $\Delta \mathrm{SpO}_{2}$ and $\mathrm{VO}_{2} \max$ $(r=-.45$, moderate effect, $p=.017)$. In addition, the mean $\mathrm{VO}_{2} \max$ values were significantly $(p=.017$, $d=0.97$, moderate effect, two-sample $t$-test) greater in $\mathrm{SG}\left(62.4 \pm 7.2 \mathrm{ml} \cdot \mathrm{kg}^{-1} \cdot \mathrm{min}^{-1}\right)$ compared with $\mathrm{RG}$ $\left(55.5 \pm 7.1 \mathrm{ml} \cdot \mathrm{kg}^{-1} \cdot \mathrm{min}^{-1}\right)$. Upon examination of the changes between the hypoxia and the preliminary 

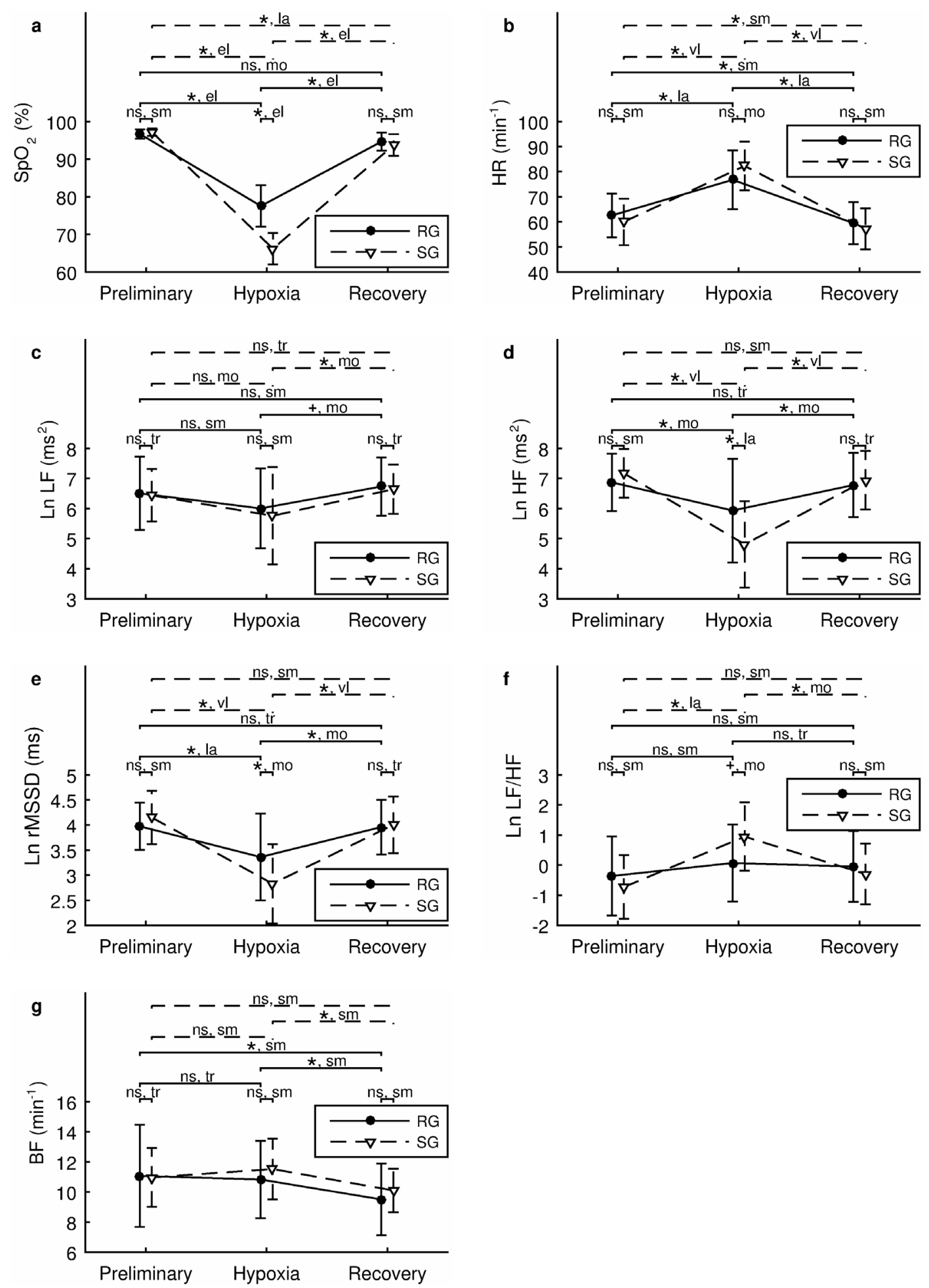

Figure 2. Changes in the oxygen saturation (a), heart rate (b), natural logarithm of low frequency power (c), natural logarithm of high-frequency power (d), natural logarithm of square root of the mean of the squares of the successive differences (e), natural logarithm of low-frequency/high-frequency ratio (f) and breath frequency (g) during Preliminary, Hypoxia, and Recovery phases. Values are presented as the mean \pm standard deviation. Comparisons by means of Fisher's LSD post-hoc test are presented. RG = resistant group (saturation during hypoxia $\geq 70.9 \%, n=14$ ); $\mathrm{SG}=$ sensitive group (saturation during hypoxia $<70.9 \%, n=14$ ); ${ }^{*}=p<.05$; $+=.05 \leq p<.06 ; \mathrm{ns}=$ nonsignificant; $\mathrm{tr}=$ trivial effect size $(|d|<0.2) ; \mathrm{sm}=$ small effect size $(|d| \geq 0.2)$; mo = moderate effect size $(|d| \geq 0.6)$; la = large effect size $(|d| \geq 1.2)$; vl = very large effect size $(|d| \geq 2.0)$; el = extremely large effect size $(|d| \geq 4.0)$. 
Table 1

Means and $S D S$ of $S p O, H R, H R V$ variables, and BF during Preliminary, Hypoxia, and Recovery phases

\begin{tabular}{|c|c|c|c|c|c|c|}
\hline \multirow[b]{2}{*}{ Variable } & \multicolumn{2}{|c|}{ Preliminary } & \multicolumn{2}{|c|}{ Hypoxia } & \multicolumn{2}{|c|}{ Recovery } \\
\hline & $\mathrm{RG}$ & $\mathrm{SG}$ & $\mathrm{RG}$ & SG & RG & SG \\
\hline $\mathrm{SpO}_{2}(\%)$ & $96.6 \pm 1.3$ & $97.1 \pm 1.0$ & $77.5 \pm 5.5$ & $66.2 \pm 4.1$ & $94.7 \pm 2.4$ & $93.7 \pm 2.9$ \\
\hline HR (beats/min) & $62.5 \pm 8.7$ & $60.0 \pm 9.3$ & $76.7 \pm 11.7$ & $82.2 \pm 9.7$ & $59.4 \pm 8.4$ & $57.2 \pm 8.2$ \\
\hline $\mathrm{LF}\left(\mathrm{ms}^{2}\right)$ & $1397 \pm 2345$ & $899 \pm 896$ & $1039 \pm 1641$ & $982 \pm 1676$ & $1205 \pm 941$ & $1076 \pm 1104$ \\
\hline $\mathrm{HF}\left(\mathrm{ms}^{2}\right)$ & $1404 \pm 1235$ & $1689 \pm 1292$ & $1095 \pm 1497$ & $428 \pm 1005$ & $1498 \pm 1674$ & $1573 \pm 1537$ \\
\hline rMSSD (ms) & $58.8 \pm 27.5$ & $71.5 \pm 35.2$ & $40.2 \pm 34.0$ & $24.8 \pm 31.0$ & $60.3 \pm 35.0$ & $63.1 \pm 34.2$ \\
\hline $\mathrm{LF} / \mathrm{HF}$ & $1.7 \pm 2.7$ & $0.8 \pm 0.7$ & $2.3 \pm 2.8$ & $4.6 \pm 6.9$ & $1.8 \pm 2.3$ & $1.1 \pm 1.1$ \\
\hline $\operatorname{Ln} \operatorname{LF}\left(\mathrm{ms}^{2}\right)$ & $6.5 \pm 1.2$ & $6.4 \pm 0.9$ & $6.0 \pm 1.3$ & $5.8 \pm 1.6$ & $6.7 \pm 1.0$ & $6.6 \pm 0.8$ \\
\hline Ln HF $\left(\mathrm{ms}^{2}\right)$ & $6.9 \pm 1.0$ & $7.2 \pm 0.8$ & $5.9 \pm 1.7$ & $4.8 \pm 1.4$ & $6.8 \pm 1.1$ & $6.9 \pm 1.0$ \\
\hline Ln rMSSD (ms) & $4.0 \pm 0.5$ & $4.1 \pm 0.5$ & $3.4 \pm 0.9$ & $2.8 \pm 0.8$ & $4.0 \pm 0.5$ & $4.0 \pm 0.6$ \\
\hline Ln LF/HF & $-0.4 \pm 1.3$ & $-0.7 \pm 1.1$ & $0.1 \pm 1.3$ & $0.9 \pm 1.1$ & $0.0 \pm 1,2$ & $-0.3 \pm 1.0$ \\
\hline $\mathrm{BF}$ & $11.1 \pm 3.4$ & $11.0 \pm 2.0$ & $10.8 \pm 2.6$ & $11.5 \pm 2.0$ & $9.5 \pm 2.4$ & $10.1 \pm 1.4$ \\
\hline
\end{tabular}

Note. $\mathrm{RG}=$ resistant group (saturation during hypoxia $\geq 70.9 \%, n=14$ ); $\mathrm{SG}=$ sensitive group (saturation during hypoxia $<70.9 \%$, $n=14) ; \mathrm{SpO}_{2}=$ arterial oxygen saturation; $\mathrm{HR}=$ heart rate; $\mathrm{LF}=$ low-frequency power; $\mathrm{HF}=$ high-frequency power; $\mathrm{rMSSD}=$ square root of the mean of the squares of the successive differences; $\mathrm{LF} / \mathrm{HF}=$ low-frequency/high-frequency ratio; $\mathrm{Ln}=$ natural logarithm; $\mathrm{BF}=$ breathing frequency.

phases (Figure 4), there were significant $(p=.017$, $d=0.97$, moderate effect, two-sample $t$-test) differences in $\Delta \mathrm{Ln} \mathrm{LF} / \mathrm{HF}$ between HDG $(1.7 \pm 1.5)$ and HCG $(0.5 \pm 0.9)$. There was also a significant difference $(p=.027, d=-0.88$, moderate effect, two-sample $t$-test $)$ in $\triangle \mathrm{SpO}_{2}$ between HDG $(-28.2 \pm 7.3 \%)$ and $\mathrm{HCG}$ $(-21.9 \pm 6.8 \%)$. However, there was insignificant difference ( $p=.366, d=0.35$, small effect, two-sample $t$-test) in $\mathrm{VO}_{2} \mathrm{max}$ between $\mathrm{HDG}\left(60.3 \pm 7.6 \mathrm{ml} \cdot \mathrm{kg}^{-1} \cdot \mathrm{min}^{-1}\right)$ and HCG $\left(57.6 \pm 8.1 \mathrm{ml} \cdot \mathrm{kg}^{-1} \cdot \mathrm{min}^{-1}\right)$.

\section{Discussion}

This study was primarily designed to assess $\mathrm{SpO}_{2}$ and autonomic cardiac regulation responses to short-term exposure to normobaric hypoxia $\left(\mathrm{FiO}_{2}=9.6 \%\right)$, and consequently to verify, whether normoxic $\mathrm{VO}_{2} \max$ level may be related to the $\mathrm{SpO}_{2}$ response to hypoxia under resting conditions. In relation to the $\mathrm{SpO}_{2}$ and HRV response to hypoxia, we also tested, whether 10 minutes of hypoxia have an impact of subjective ratings of perceived comfort/discomfort.

The main findings of the study were a) during hypoxia, SG showed a greater decline in vagal activity compared with RG, with a relative increase in sympathetic activity apparent in SG only; b) there was a negative correlation $(r=-.45, p=.017)$ between normoxic $\mathrm{VO}_{2}$ max level and $\Delta \mathrm{SpO}_{2}$ in hypoxia; c) HDG demonstrated a greater reduction in $\mathrm{SpO}_{2}$ with a relative increase in sympathetic activity in hypoxia compared with HCG; d) Ln rMSSD exhibited a stronger association $(r=.64 ; p<.001)$ with $\mathrm{SpO}_{2}$ during hypoxia compared with Ln HF $(r=.54 ; p=.003)$.

Our results demonstrated that $\mathrm{SpO}_{2}$ was reduced during hypoxia in both $\mathrm{RG}$ and $\mathrm{SG}$, with the decrease in $\mathrm{SpO}_{2}$ level significantly greater in $\mathrm{SG}$, where $\mathrm{SG}$ demonstrated a significantly greater $\mathrm{VO}_{2}$ max compared with RG. In addition, a correlation $(r=-.45, p=.017)$ was found between normoxic $\mathrm{VO}_{2}$ max and the $\mathrm{SpO}_{2}$ response during acute exposure to normobaric hypoxia at rest. From this finding, it appears that individuals with greater cardiorespiratory fitness may be more sensitive to an acute reduction in oxygen delivery to tissues at rest compared with individuals who have a lower $\mathrm{VO}_{2}$ max. Based on this result, one would hypothesize that $\mathrm{VO}_{2}$ max level may be considered as an indicator (predictor) of $\mathrm{SpO}_{2}$ response to hypoxia exposure in previously non-acclimatized subjects.

Our findings are supported by Woorons et al. (2007) who reported that both greater arterial desaturation and lower end-tidal oxygen pressure was found in endurance trained compared with untrained subjects during 5 minutes rest, and following moderate exercise, while subjects experienced normobaric hypoxia $\left(\mathrm{FiO}_{2}=15.4\right.$ to $\left.11.7 \%\right)$. These authors suggested that a decline in arterial desaturation at rest, but particularly during moderate exercise, due to a blunted chemoreceptor sensitivity to hypoxia, resulting in an insufficient ventilatory response (a relative hypoventilation) to severe hypoxia in trained subjects. Botek et al. (2015) investigated the $\mathrm{SpO}_{2}$ and $\mathrm{HRV}$ response to normobaric hypoxia in health males, and observed a return of $\mathrm{SpO}_{2}$ to pre-hypoxia level within 5 minutes 

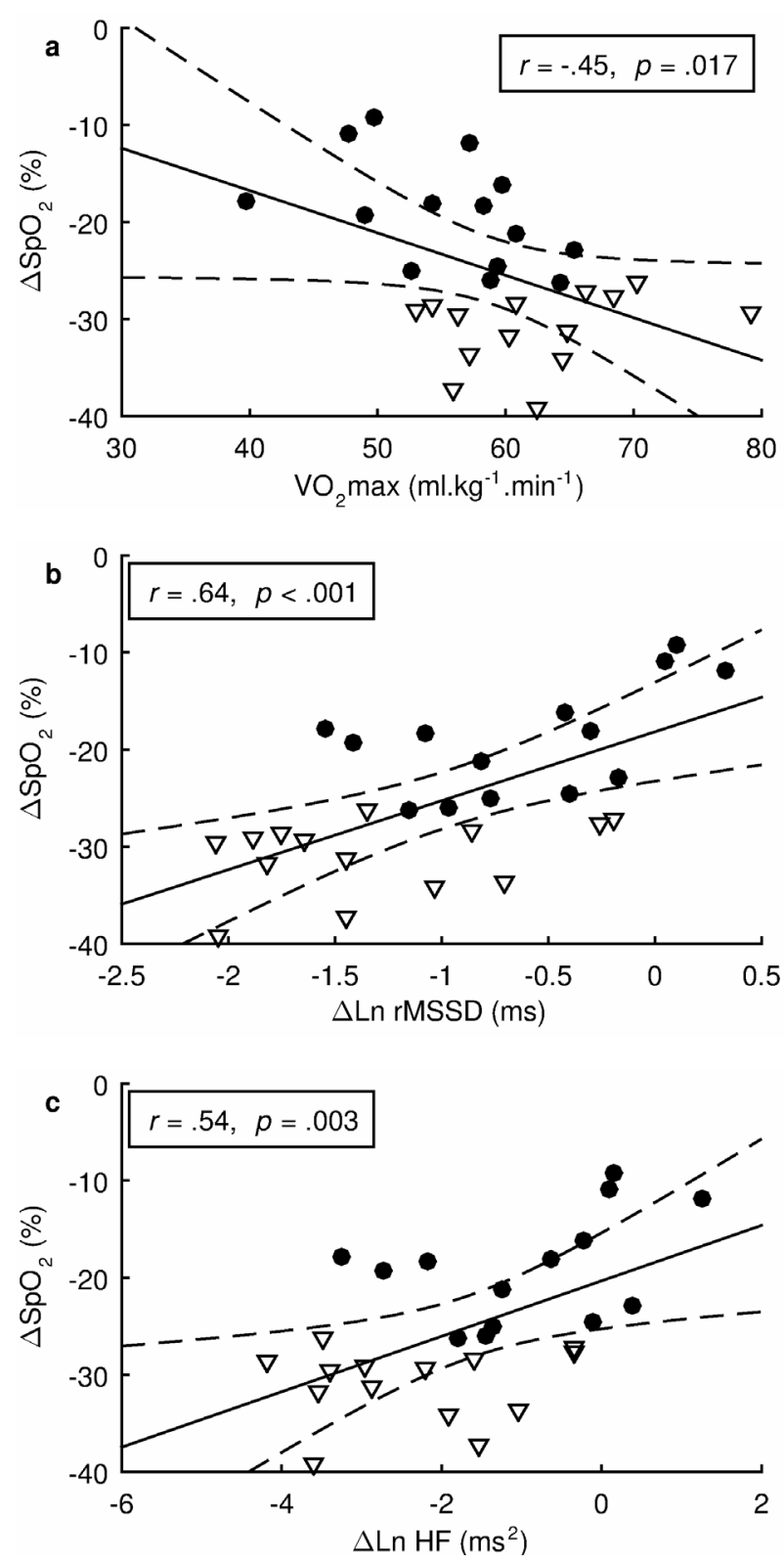

Figure 3. Correlation analysis between oxygen desaturation $\left(\Delta \mathrm{SpO}_{2}=\mathrm{SpO}_{2 \text { Hypoxia }}-\mathrm{SpO}_{2 \text { Preliminary }}\right)$ and the $\mathrm{VO}_{2}$ max value (a), the change in the natural logarithm of square root of the mean of the squares of the successive differences (b, $\Delta$ Ln rMSSD $=$ Ln rMSSD ${ }_{\text {Hypoxia }}-$ Ln rMSSD $_{\text {Preliminary }}$ ), and the change in the natural logarithm of high-frequency power (c, $\Delta \mathrm{Ln} \mathrm{HF}=\mathrm{Ln} \mathrm{HF}_{\text {Hypoxia }}-\mathrm{Ln}$ $\left.\mathrm{HF}_{\text {Preliminary }}\right)$. Circle mark $=$ resistant group (saturation during hypoxia $\geq 70.9 \%, n=14$ ); triangle mark = sensitive group (saturation during hypoxia $<70.9 \%, n=14$ ). Dashed lines denote 0.95 -confidence interval.

of recovery with no differences between SG and RG groups using paced breathing at 12 breaths per minute. Similarly, our results showed no differences between groups, while $\mathrm{SpO}_{2}$ in $\mathrm{SG}$ was delayed in returning to the baseline level when $\mathrm{BF}$ was at 10 breaths per minute. Thus, it seems that a lower BF in the present
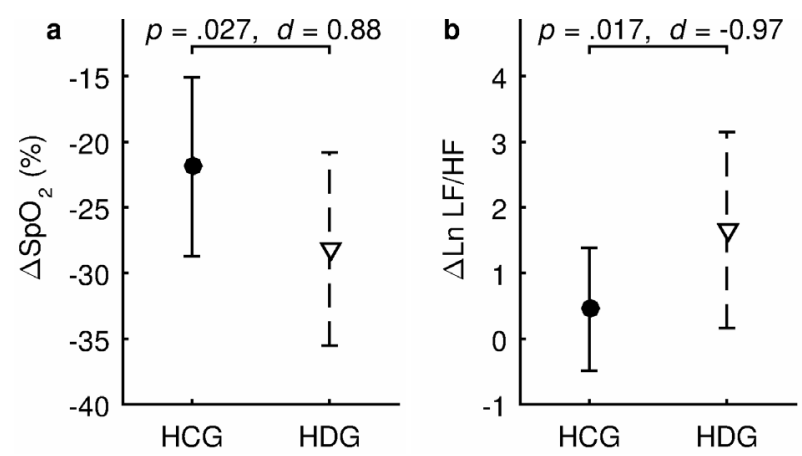

Figure 4. Differences in the oxygen desaturation (a, $\Delta \mathrm{SpO}_{2}=\mathrm{SpO}_{2 \text { Hypoxia }}-\mathrm{SpO}_{2 \text { Preliminary }}$ ) and natural logarithm of low-frequency/high-frequency ratio change (b, $\left.\Delta \mathrm{Ln} \mathrm{LF} / \mathrm{HF}=\mathrm{Ln} \mathrm{LF} / \mathrm{HF}_{\text {Hypoxia }}-\mathrm{Ln} \mathrm{LF} / \mathrm{HF}_{\text {Preliminary }}\right)$ between hypoxia comfort group (HCG, $n=14$ ) and hypoxia discomfort group (HDG, $n$ 14). Values are presented as the mean \pm standard deviation. The comparison by means of two-sample t-test is presented.

study may have possibly contributed to the incomplete recover of $\mathrm{SpO}_{2}$ after hypoxia exposure.

Regarding HRV, we found insignificant and small changes in the sympathovagal balance (LF/HF ratio) across the three experimental phases in RG. Similar results for $\mathrm{LF} / \mathrm{HF}$ were published previously (Bobyleva \& Glazachev, 2007; Botek et al., 2015). A significant reduction in vagal activity ( $\mathrm{Ln} \mathrm{HF}$ ) occurred during the hypoxia period compared with the preliminary phase in both RG (moderate effect) and SG (very large effect). However, SG demonstrated a significantly (large effect) greater withdrawal in vagal activity compared with RG. The different vagal activity responses to hypoxia between groups resulted in greater shift in sympathovagal balance towards sympathetic side in the SG compared with RG. The difference in sympathovagal balance (Ln LF/HF) during hypoxia between SG and RG was moderate but only approaching significance $(p=.052)$. Based on this result, it is tenable that differences in autonomic cardiac disturbances during acute normobaric hypoxia could be partly attributed to $\mathrm{VO}_{2}$ max level. Bobyleva and Glazachev (2007) demonstrated that hypoxia-sensitive subjects undergoing intermittent hypoxic training (IHT) (20 sessions of 60 minutes) had a blunted sympathetic response and a slower decline in $\mathrm{SpO}_{2}$ during hypoxia. Therefore, future research is needed to clarify the effect of IHT on $\mathrm{SpO}_{2}$ and ANS response in aerobic well trained subjects that in our study exhibit both autonomic cardiac impairment and a greater $\mathrm{SpO}_{2}$ change during acute hypoxia.

During the hypoxic phase, Ln rMSSD, as a time domain index of vagal activity (Buchheit, 2014), was significantly and at least largely reduced compared with the preliminary phase in both groups. Moreover, 
Ln $\operatorname{rMSSD}(r=.64, p<.001)$ was closely related to the $\mathrm{SpO}_{2}$ level during hypoxia compared with $\mathrm{Ln} \mathrm{HF}$ power $(r=.54, p=.003)$. A possible reason for this finding may be because of six subjects whose BF was under 9 breaths per minute during the hypoxic phase. The result of this was previously described (GarcíaGonzález, Vázquez-Seisdedos, \& Pallàs-Areny, 2000; Vlčková et al., 2005) by an artificial increase in both LF and LF/HF ratio due to RSA peak shift towards the LF. Based on these results, it seems that rMSSD is a more robust index of vagal activity in normobaric hypoxia than HF when using spontaneous breathing.

There is an increasing number of travellers who journey to high altitudes $(>2400 \mathrm{~m})$, where there is increased risk of AMS development (Gallagher \& Hackett, 2004). It has previously been shown that both reduced $\mathrm{SpO}_{2}$ (Burtscher et al., 2004; Karinen et al., 2010) and a stimulated sympathetic system (Mazzeo et al., 1995) during hypoxia contributes to AMS susceptibility. Our findings demonstrate a significantly greater desaturation level in subjects who rated their 10 minute hypoxia experience as mild or moderately uncomfortable, because of the presence of nausea or vomiting, headache, and weakness, while subjects free of perceived hypoxia-induced side effects demonstrated a significantly lower desaturation together with a lower relative increase in sympathetic activity. In a previous study it was reported that measurement of the $\mathrm{SpO}_{2}$ responses to hypoxia $\left(\mathrm{FiO}_{2}=11.5 \%\right)$ at rest and during 5 minute exercise $\left(50 \%\right.$ of normoxic $\mathrm{VO}_{2} \max$ ) allows the detection of those subjects who are more liable to suffer from AMS (Rathat et al., 1992). It was also suggested that the monitoring of the $\mathrm{SpO}_{2}$ response to hypobaric or normobaric hypoxia represents a useful method for detecting subjects who are highly susceptible to AMS (Burtscher et al., 2004). However, in contrast, Rathat et al. (1992) reported that subjects should be exposed to hypoxia in resting state of at least 20-30 minutes to identify risk of AMS. Based on the previous literature (Burtscher et al., 2004; Rathat et al., 1992) and our findings, we feel that a controlled exposure to simulated altitude equal to $\sim 6200 \mathrm{~m}$ for 10 minutes in unacclimatized persons before the travelling to high altitude may serve as the screening measurement to assess an individual's hypoxia tolerance. Susceptibility to AMS was, in addition to a reduction in $\mathrm{SpO}_{2}$, also associated with better aerobic capacity, younger age, and higher body mass index in mountain climbers (Karinen et al., 2010). However, we did not find a significant difference in $\mathrm{VO}_{2}$ max between $\mathrm{HCG}$ and HDG in contrast to Karinen et al. (2010). A discrepancy in results may be explained by the difference in study design. In our study, volunteers were exposed to normobaric hypoxia for 10 minutes during rest, whereas Karinen et al. (2010) assessed $\mathrm{SpO}_{2}$ response in climbers during mountain expeditions.

A limitation of this study was that subjects wore a face mask, and therefore, it was not possible to assess changes in the ventilatory response that may have occurred during the phases of the study. Therefore, the HRV diagnostics system (DiANS PF8) was used to evaluate the changes in BF. Future research should include ventilatory response monitoring and the continual monitoring of blood pressure that may be beneficial for providing a more complex view of the transport system response during acute hypoxia.

\section{Conclusion}

The results demonstrated that a $\mathrm{SpO}_{2}$ reduction increases perceived hypoxia discomfort, which is accompanied by a greater withdrawal in cardiac vagal activity. In addition, it was shown that 10 minutes of hypoxia exposure induced a greater reduction in $\mathrm{SpO}_{2}$ and autonomic cardiac disturbance in individuals with greater normoxic $\mathrm{VO}_{2}$ max values. A practical application of this finding is that hypoxic exposure before travel to altitude may help with the screening of hypoxia-sensitive persons who may be more prone to suffer AMS, specifically in individuals with a high aerobic capacity.

\section{Acknowledgment}

This study was supported by a grant of the Faculty of Physical Culture, Palacký University Olomouc (no. IGA_FTK_2015_006).

\section{Conflict of interest}

There were no conflicts of interest.

\section{References}

Akselrod, S., Gordo, D., Ubel, F. A., Shannon, D. C., Berger, A. C., \& Cohen, R. J. (1981). Power spectrum analysis of heart rate fluctuation: A quantitative probe of beat-to-beat cardiovascular control. Science, 213, 220-222.

Al Haddad, H., Mendez-Villanueva, A., Bourdon, P. C., \& Buchheit, M. (2012). Effect of acute hypoxia on post-exercise parasympathetic reactivation in healthy men. Frontiers in Physiology, 3, 289.

Åstrand, P. O., Rodahl, K., Dahl, H. A., \& Strømme, S. B. (2003). Textbook of work physiology: Physiological bases of exercise. Champain, IL: Human Kinetics. 
Bobyleva, O. V., \& Glazachev, O. S. (2007). Changes in autonomic response and resistance to acute graded hypoxia during intermittent hypoxic training. Human Physiology, 33, 199-206.

Botek, M., Krejčí, J., De Smet, S., Gába, A., \& McKune, A. J. (2015). Heart rate variability and arterial oxygen saturation response during extreme normobaric hypoxia. Autonomic Neuroscience: Basic \& Clinical, 190, 40-45.

Brown, T. E., Beightol, L. A., Koh, J., \& Eckberg, D. L. (1993). Important influence of respiration on human R-R interval power spectra is largely ignored. Journal of Applied Physiology, 75, 2310-2317.

Buchheit, M. (2014). Monitoring training status with HR measures: Do all roads lead to Rome? Frontiers in Physiology, 27, 73.

Buchheit, M., Richard, R., Doutreleau, S., Lonsdorfer-Wolf, E., Brandenberger, G., \& Simon, C. (2004). Effect of acute hypoxia on heart rate variability at rest and during exercise. International Journal of Sports Medicine, 25, 264-269.

Burtscher, M., Flatz, M., \& Faulhaber, M. (2004). Prediction of susceptibility to acute mountain sickness by $\mathrm{SaO}_{2}$ values during short-term exposure to hypoxia. High Altitude Medicine \& Biology, 5, 335-340.

Fritz, C. O., Morris, P. E., \& Richler, J. J. (2012). Effect size estimates: Current use, calculations, and interpretation. Journal of Experimental Psychology. General, 141, 2-18.

Gallagher, S. A., \& Hackett, P. H. (2004). High-altitude illness. Emergency Medicine Clinics of North America, 22, 329-355.

García-González, M. A., Vázquez-Seisdedos, C., \& PallàsAreny, R. (2000). Variations in breathing patterns increase low frequency contents in HRV spectra. Physiological Measurement, 21, 417-423.

Hirsch, J. A., \& Bishop, B. (1981). Respiratory sinus arrhythmia in humans: How breathing pattern modulates heart rate. American Journal of Physiology, 241, H620-H629.

Hopkins, W. G., Marshall, S. W., Batterham, A. M., \& Hanin, J. (2009). Progressive statistics for studies in sports medicine and exercise science. Medicine and Science in Sports and Exercise, 41, 3-13.

Hori, K., Yamakawa, M., Tanaka, N., Murakami, H., Kaya, M., \& Hori, S. (2005). Influence of sound and light on heart rate variability. Journal of Human Ergology, 34, 25-34.

Howley, E. T., Bassett, D. R., \& Welch, H. G. (1995). Criteria for maximal oxygen uptake: Review and commentary. Medicine and Science in Sports and Exercise, 27, 1292-1301.

Iwasaki, K. I., Ogawa, Y., Aoki, K., Saitoh, T., Otsubo, A., \& Shibata, S. (2006). Cardiovascular regulation response to hypoxia during stepwise decreases from $21 \%$ to $15 \%$ inhaled oxygen. Aviation, Space, and Environmental Medicine, 77, 1015-1019.

Karinen, H. M., Peltonen, J. E., Kähönen, M., \& Tikkanen, H. O. (2010). Prediction of acute mountain sickness by monitoring arterial oxygen saturation during ascent. High Altitude Medicine \& Biology, 11, 325-332.

Malik, M., \& Camm, A. J. (1993). Components of heart rate variability - what they really mean and what we really measure. American Journal of Cardiology, 72, 821-822.

Malliani, A., Lombardi, F., \& Pagani, M. (1994). Power spectrum analysis of heart rate variability: A tool to explore neural regulatory mechanisms. British Heart Journal, 71, $1-2$.

Malliani, A., Pagani, M., Lombardi, F., \& Cerutti, S. (1991). Cardiovascular neural regulation explored in the frequency domain. Circulation, 84, 482-492.

Mazzeo, R. S., Brooks, G. A., Butterfield, G. E., Podolin, D. A., Wolfel, E. E., \& Reeves, J. T. (1995). Acclimatization to high altitude increase muscle sympathetic activity both at rest and during exercise. American Journal of Physiology, 269, R201-R207.

Midgley, A. W., McNaughton, L. R., Polman, R., \& Marchant, D. (2007). Criteria for determination of maximal oxygen uptake. Sports Medicine, 37, 1019-1028.

Millet, G. P., Libicz, S., Borrani, F., Fattori, P., Bignet, F., \& Candau, R. (2003). Effects of increased intensity of intermittent training in runners with differing $\mathrm{VO}_{2}$ kinetics. European Journal of Applied Physiology, 90, 50-57.

Millet, G. P., Roels, B., Schmitt, L., Woorons, X., \& Richalet, J. P. (2010). Combining hypoxic methods for peak performance. Sports Medicine, 40, 1-25.

Mizuno, M. J. C., Bro-Rasmussen, T., Mygind, E., Schibye, B., Rasmussen, B., \& Saltin, B. (1990). Limb skeletal muscle adaptation in athletes after training at altitude. Journal of Applied Physiology, 68, 496-502.

Morris, S. B., \& DeShon, R. P. (2002). Combining effect size estimates in meta-analysis with repeated measures and independent-groups designs. Psychological Methods, 7, 105-125.

Narkiewicz, K., Van De Borne, P., Montano, N., Hering, D., Kara, T., \& Somers, V. K. (2006). Sympathetic neural outflow and chemoreflex sensitivity are related to spontaneous breathing rate in normal men. Hypertension, 47, 51-55.

O'Connor, T., Dubowitz, G., \& Bickler, P. E. (2004). Pulse oximetry in the diagnosis of acute mountain sickness. High Altitude Medicine \& Biology, 5, 341-348.

Patwardhan, A. R., Vallurupalli, S., Evans, J. M., Bruce, E. N., \& Knapp, C. F. (1995). Override of spontaneous respiratory pattern generator reduces cardiovascular parasympathetic influence. Journal of Applied Physiology, 79, 1048-1054.

Penttilä, J., Helminen, A., Jartti, T., Kuusela, T., Huikuri, H. V., Tulppo, M. P., \& Scheinin, H. (2001). Time domain, geometrical and frequency domain analysis of cardiac vagal outflow: Effects of various respiratory patterns. Clinical Physiology, 21, 365-376.

Pighin, S., Bonini, N., Savadori, L., Hadjichristidis, C., Antonetti, T., \& Schena, F. (2012). Decision making under hypoxia: Oxygen depletion increases risk seeking for losses but not for gains. Judgment and Decision Making, 7, 472-477.

Rathat, C., Richalet, J. P., Herry, J. P., \& Larmignat, P. (1992). Detection of high-risk subjects for high altitude diseases. International Journal of Sports Medicine, 13, S76-S78.

Roach, R. C., Maes, D., Sandoval, D., Robergs, R. A., Icenogle, M., Hinghofer-Szalkay, H., ... Loeppky, J. A. (2000). Exercise exacerbates acute mountain sickness at simulated high altitude. Journal of Applied Physiology, 88, 581-585.

Roche, F., Reynaud, C., Garet, M., Pichot, V., Costes, F., \& Barthélémy, J. C. (2002). Cardiac baroreflex control in humans during and immediately after brief exposure to 
simulated high altitude. Clinical Physiology and Functional Imaging, 22, 301-306.

Salinger, J., Štěpaník, P., Kolisko, P., Stejskal, P., Theuerová, Š., Elfmark, M., ... Krejčí, J. (2005). Measurement of breathing frequency from ECG in the examination of autonomous nervous system activities: Suggested methods and their verification. Acta Universitatis Palackianae Olomucensis. Gymnica, 35(2), 95-103.

Sasaki, K., \& Maruyama, R. (2014). Consciously controlled breathing decreases the high-frequency component of heart rate variability by inhibiting cardiac parasympathetic nerve activity. Tohoku Journal of Experimental Medicine, 233, 155-163.

Task Force of the European Society of Cardiology and the North American Society of Pacing and Electrophysiology. (1996). Heart rate variability: Standards of measurement, physiological interpretation and clinical use. Circulation, 93, 354-381.

Ursino, M., \& Magosso, E. (2003). Role of short-term cardiovascular regulation in heart period variability: A modeling study. American Journal of Physiology, 284, H1479-H1493.

Ventura, N., Hoppeler, H., Seiler, R., Binggeli, A., Mullis, P., \& Vogt, M. (2003). The response of trained athletes to six weeks of endurance training in hypoxia or normoxia. International Journal of Sports Medicine, 24, 166-172.

Vlčková, E., Stejskal, P., Jakubec, A., Řehová, I., Pavlík, F., \& Botek, M. (2005). Controlled breathing and heart rate variability. Medicina Sportiva, 9, 28-32.

Wille, M., Mairer, K., Gatterer, H., Philippe, M., Faulhaber, M., \& Burtscher, M. (2012). Changes in cardiac autonomic activity during a passive 8 hour acute exposure to 5 $500 \mathrm{~m}$ normobaric hypoxia are not related to the development of acute mountain sickness. International Journal of Sports Medicine, 33, 186-191.

Woorons, X., Mollard, P., Pichon, A., Lamberto, C., Duvallet, A., \& Richalet, J. P. (2007). Moderate exercise in hypoxia induces a greater arterial desaturation in trained than untrained men. Scandinavian Journal of Medicine \& Science in Sports, 17, 431-436.

Yasuma, F., \& Hayano, J. (2004). Respiratory sinus arrhythmia: Why does the heartbeat synchronize with respiratory rhythm? Chest, 125, 683-690.

Zygmunt, A., \& Stanczyk, J. (2010). Methods of evaluation of autonomic nervous system function. Archives of Medical Science, 6, 11-18. 